\title{
Rheological and Thermal Transport Characteristics of a Transformer Oil Based Ferrofluid
}

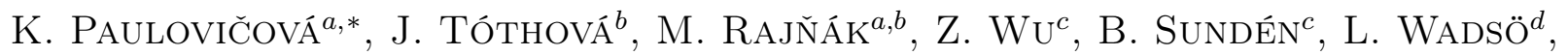 \\ T. TOBIÁS̆ $\check{S}^{a}, \mathrm{P}$. KOPČANSKÝ ${ }^{a}$, M. TIMKO ${ }^{a}$ AND V. LISÝ ${ }^{b}$ \\ ${ }^{a}$ Institute of Experimental Physics, Slovak Academy of Sciences, Watsonova 47, Košice, Slovakia \\ ${ }^{b}$ Faculty of Electrical Engineering and Informatics, Technical University of Košice, Letná 9, Košice, Slovakia \\ ${ }^{c}$ Department of Energy Sciences, Lund University, 22100 Lund, Sweden \\ ${ }^{d}$ Division of Building Materials, Lund University, 22100 Lund, Sweden
}

\begin{abstract}
Ferrofluids based on insulating liquids are intensively studied as a potential substitute of liquid dielectric in high voltage technologies. In this work we focus on the experimental investigation of flow and thermal transport characteristics of a ferrofluid based on transformer oil (Mogul) and iron oxide nanoparticles. The magneto-rheological behavior of the ferrofluid was studied by a rotational rheometer in the shear rate range from 1 to $1000 \mathrm{~s}^{-1}$ and magnetic field up to $1 \mathrm{~T}$. By means of a thermal constants analyzer and a transient plane source method we obtained the thermal conductivity, specific heat and thermal diffusivity values for the studied oil and the ferrofluid. It is shown that the Newtonian character of the ferrofluid changes to a non-Newtonian with application of the magnetic field. The notable magneto-viscous effect has been observed especially at low shear rates. We found that the doping of the transformer oil by $3 \mathrm{wt} \%$ of the nanoparticles results in a thermal conductivity enhancement by about $3.2 \%$.
\end{abstract}

DOI: 10.12693 /APhysPolA.133.564

PACS/topics: 47.65.Cb, 83.80.Gv, 66.20.Ej, 44.35.+c

\section{Introduction}

The transformer oil-based nanofluids with improved dielectric and thermal properties have the potential to replace mineral oil base products in high voltage technologies. They are advantageous because they function more superior than standard insulating liquid materials such as mineral oil and they contribute to complete insulating and thermal gains. A transformer, which transforms voltage and transfers energy, is the most critical part of an electric power network. Most of the transformers in operation around the world are close to or beyond their designed life time. The typical service life time of transformers which failed because of insulation problems is 17.8 years, and $75 \%$ of high voltage transformers collapses originated in dielectric insulation failure [1]. The operational reliability and lifetime of transformers primarily rely on traits and status of insulation material. Due to the increasing demand of high voltage rate and small size for transformers, the development of transformer oils with favorable dielectric and thermal characteristics are extensively required. Ferrofluids, the well-known and prominent term nowadays in the dielectric society, have been the subject of huge research work over the past decade $[1,2]$. A liquid with nanosized superparamagnetic particles homogeneously suspended in a nonmagnetic carrier liquid at just a few weight percentage (wt\%) is called a ferrofluid (FF) or a magnetic

\footnotetext{
*corresponding author; e-mail: paulovic@saske.sk
}

fluids. Brownian motion keeps the nanoscopic particles from settling under gravity, and a surfactant layer, such as oleic acid, surrounds each particle to provide short range steric hindrance between particles, preventing particle agglomeration. These nanofluids are a kind of smart materials (also called active materials), whose physical properties can be controlled and by external magnetic or electric field. In consequence of the magnetic dipolar-dipolar interaction between the magnetic particles, the magnetic fluids demonstrate typical magnetorheological (MR) effects, as their viscosity could change reversibly and rapidly by tuning the external magnetic field (MF) [2-4]. Thus, magnetic particles play the key role in determining the MR effect for the magnetic fluid. During the application of the MF, the magnetic particles assemble to form chain-like structures, thus increasing the viscosity $[4,5]$. Besides the rheological investigation of FFs, intensive research has been conducted on their thermal transport properties too [5-9].

In this paper we focus on the experimental investigation of magneto-rheological and thermal transport properties of a FF based on new and commercially available transformer oil containing iron oxide nanoparticles.

\section{Experimental}

The studied FF consisted of transformer oil Mogul Trafo CZ-A and $\mathrm{Fe}_{3} \mathrm{O}_{4}$ nanoparticles synthesized by a chemical co-precipitation method and sterically stabilized by a monolayer of oleic acid in order to prevent particles agglomeration. The magnetic particles diameter determined from a transmission electron microscopy was 
found to be $10 \mathrm{~nm}$. The micrographies also show a shape of the particles very close to the spherical one (Fig. 1). The density of the FF was determined to be $0.881 \mathrm{~g} \mathrm{~cm}^{-3}$. From magnetization measurements performed by a vibrating sample magnetometer (VSM) method, the magnetic mass fraction of the prepared FF was defined as $3 \%$ and the nanoparticles exhibited superparamagnetic behavior at room temperature.

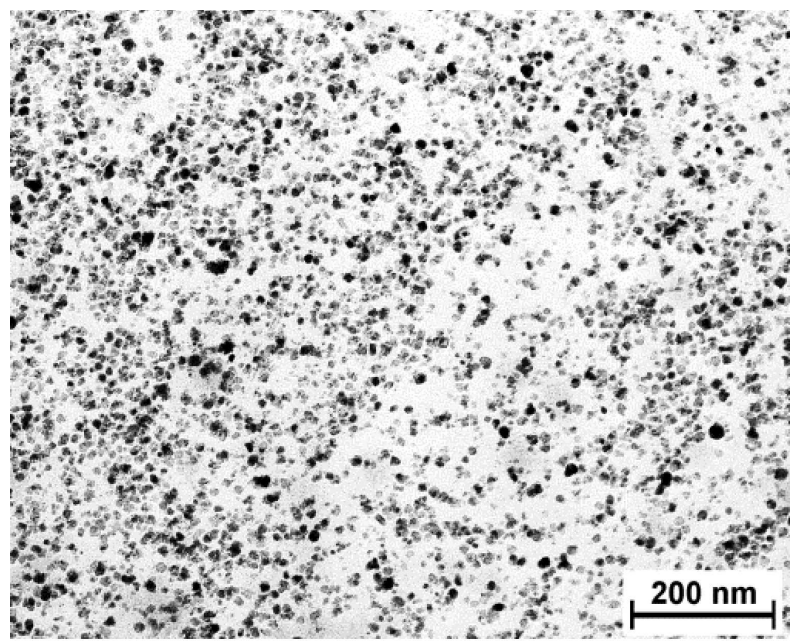

Fig. 1. Transmission electron microscope image of the synthesized magnetic nanoparticles.

The viscosity of the FF was investigated by using a commercial rheometer (Physica MCR 502, Anton Paar Co., Austria). The measuring geometry was a parallel plates (diameter $16 \mathrm{~mm}$ ) arrangement. The MF was produced by applying an electrical current to a coil below the bottom plate. A two part magnetic cover serves as a magnetic bridge and ensures a uniform MF oriented perpendicularly to the measuring gap. The volume of $0.3 \mathrm{~cm}^{3}$ of the testing sample was used. The gap between the plates was kept at $0.328 \mathrm{~mm}$. The viscosity of the FF was measured at temperature of $20^{\circ} \mathrm{C}$. Magnetic flux density, controlled by a separate power supply unit, was varied from $0 \mathrm{mT}$ to $1000 \mathrm{mT}$. The rheological curves were obtained in shear rate sweep tests by changing the shear rate in the interval from 1 to $1000 \mathrm{~s}^{-1}$, while keeping the magnetic flux density as a constant.

The basic thermal transport properties such as the thermal conductivity, specific heat and thermal diffusivity of the transformer oil and the FF were studied by a thermal constants analyzer (TPS 2500S from Hot Disk AB, Sweden) using a transient plane source method (TPS). The sensor was inserted vertically into a small container with liquid samples to avoid the risk forbubbles.

\section{Rheological and thermal transport properties of the studied transformer oil based ferrofluid}

The viscosity of the used transformer oil was investigated in the temperature range from $20^{\circ} \mathrm{C}$ to $70^{\circ} \mathrm{C}$ as a function of the shear rate in the interval from 1 to $1000 \mathrm{~s}^{-1}$. The rheological behavior of the carrier liquid demonstrated that this transformer oil is a typical Newtonian fluid as the shear stress increases linearly with the shear rate. Experimental data showed a classical behavior, at which increasing temperature initiates decreasing viscosity of the Mogul Trafo CZ-A. The viscosity of the $\mathrm{FF}$ was measured in the presence of $\mathrm{MF}$ in the range from $0 \mathrm{mT}$ to $1000 \mathrm{mT}$ at $20^{\circ} \mathrm{C}$. The transformer oil containing the superparamagnetic nanoparticles shows the magnetoviscous (MV) effect, especially at low shear rates as shown in Fig. 2. At low shear rate $\left(1 \mathrm{~s}^{-1}\right)$, under the influence of MF, the particles interact with the MF and with each other and form a chain structure aligned in the direction of the field.

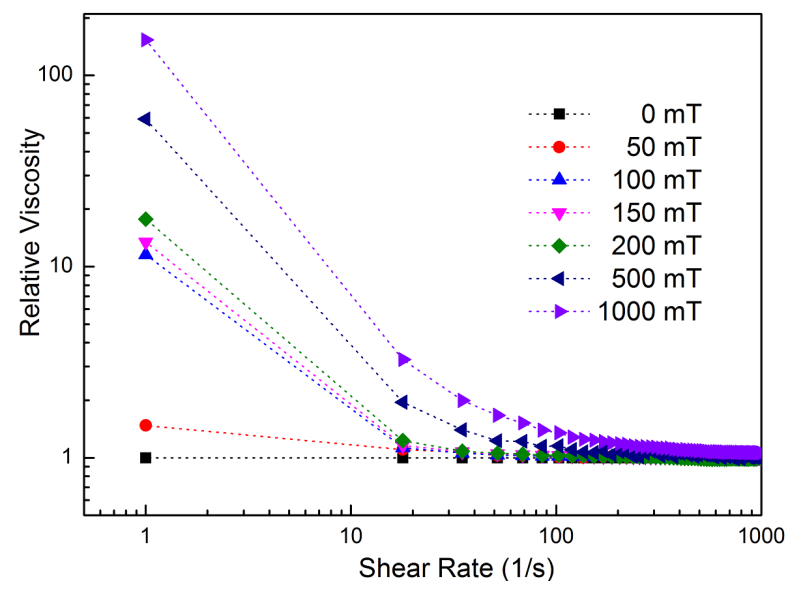

Fig. 2. Magnetoviscous effect in the studied magnetic nanofluid exposed to various magnetic field intensities.

Therefore, the FF exhibits a higher MV effect due to strong dipolar interactions. In the middle shear rate regime $\left(18-100 \mathrm{~s}^{-1}\right)$, the FF shows shear thinning effect, where viscosity decreases with shear rate due to the dominance of the hydrodynamic force $\left(F_{H}\right)$ over magnetostatic force $\left(F_{M}\right)$, which leads to a decrease in MV effect. At the shear rate regime $\left(100-1000 \mathrm{~s}^{-1}\right)$, the chainlike structures become destroyed and thereby due to the presence of individual magnetic particles and strong $F_{H}$, the MV effect diminishes.

Figure 3 confirms the assumed behavior of FF at which the relative viscosity, $\eta / \eta_{0}$ ( $\eta$ and $\eta_{0}$ are the viscosities of $\mathrm{FF}$ in the presence and in the absence of MF, respectively), increases with increasing MF.

By means of the standardized TPS technique, the thermal conductivity $\sigma$, thermal diffusivity $\tau$ and specific heat $c$ were determined simultaneously from a single measurement. Before the measurements of the transformer oil and the investigated FF, water was tested to check the accuracy of the TPS method. At room temperature $\left(21.5^{\circ} \mathrm{C}\right)$, the measured value was $0.5995 \mathrm{~W} /(\mathrm{mK})$, averaged over ten runs, with a standard deviation less than $1.0 \%$. The literature value for thermal conductivity of water at $21.5^{\circ} \mathrm{C}$ from NIST is $0.6010 \mathrm{~W} /(\mathrm{mK})$. Thus, 


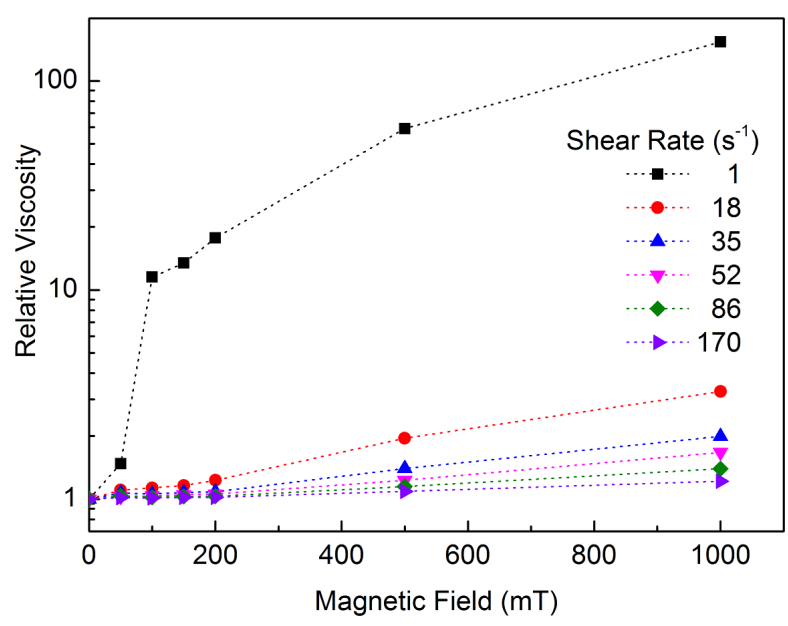

Fig. 3. The relative viscosity of FF versus increasing magnetic field at different shear rate.

the measurement uncertainty of the thermal conductivity is less than $1.0 \%$. The uncertainties of specific heat and thermal diffusivity are expected to be less than $5.0 \%$. Then, the thermal conductivity, specific heat and thermal diffusivity values of the transformer oil and the FF were measured. The obtained values are listed in Table I. It is clear that the thermal conductivity of the transformer oil, which is a crucial parameter for a cooling medium, has been enhanced by doping the transformer oil with magnetic nanoparticles by about $3.2 \%$. Similarly, the thermal diffusivity of the FF has been increased by about $13.4 \%$, while the specific heat has been decreased by about 10.1 $\%$ as compared to the values for the pure transformer oil. The enhanced thermal transport properties of the studied FF are associated with the large surface area of the nanoparticles which allows for more heat transfer. Besides the effective thermal interaction of the nanoparticles, the high mobility of the nanoparticles, attributable to the nanosize, leads to the enhanced thermal transport properties too. The particle movement may bring about micro-convection of the FF and hence increase the heat transfer, as it is typical for nanofluids in general [10].

TABLE I

Measured thermal transport properties averaged over ten runs at $21.5{ }^{\circ} \mathrm{C}$.

\begin{tabular}{c|c|c|c}
\hline \hline Sample & $\sigma[\mathrm{W} /(\mathrm{mK})]$ & $\tau[\mathrm{kJ} /(\mathrm{kgK})]$ & $c\left[\mathrm{~mm}^{2} / \mathrm{s}\right]$ \\
\hline water & 0.5995 & 4.332 & 0.1396 \\
transformer oil & 0.1679 & 1.511 & 0.1345 \\
ferrofluid & 0.1734 & 1.359 & 0.1553
\end{tabular}

\section{Conclusions}

In this study we focused on the experimental investigation of flow and thermal transport characteristics, and magneto-rheological behavior of a FF based on transformer oil Mogul Trafo CZ-A. It has been proven that the transformer oil exhibits a typical Newtonian behavior. On the other hand, the prepared FF showed the properties of a non-Newtonian fluid when exposed to the static magnetic field, as its viscosity is remarkably dependent on the shear rate. Thus, the measurements confirmed the expected magnetoviscous effect, especially at the low shear rates. Based on the thermal transport measurements, it was found that the presence of the magnetic nanoparticles in the oil (3\%) increases its thermal conductivity by about $3.2 \%$. It can be therefore concluded, that the prepared FF could find a potential application as an efficient cooling liquid medium in electrical devices like transformers or circuit breakers, as the low particle concentration is expected to have no deteriorative effect on the insulating characteristics of the transformer oil.

\section{Acknowledgments}

This work was supported by the Slovak Academy of Sciences and Ministry of Education in the framework of projects VEGA Nos. 2/0016/17, 2/0141/16, 1/0348/15 and $1 / 0311 / 15$, Ministry of Education Agency for structural funds of EU Project: ITMS 26220220061 and Slovak Research and Development Agency under the Contract No. APVV-15-0453 (M-Vision).

\section{References}

[1] M. Rafiq, Y. Lv, Ch. Li, J. Nanomater. 2016, (2016).

[2] M. Rajnak, M. Timko, P. Kopcansky, K. Paulovicova, J. Tothova, J. Kurimsky, B. Dolnik, R. Cimbala, M.V. Avdeev, V.I. Petrenko, A. Feoktystov, J. Magn. Magn. Mater. 431, 99 (2017).

[3] J. Wu, L. Pei, S. Xuan, Q. Yan, X. Gong, J. Magn. Magn. Mater. 408, 18 (2016).

[4] X. Ruan, L. Pei, S. Xuan, Q. Yan, X. Gong, J. Magn. Magn. Mater. 429, 1 (2017).

[5] J. Kúdelčík, P. Bury, Š. Hardoň, M. Sedlačík, M. Mrlík, in: 2016 ELEKTRO Strbske Pleso, IEEE, 2016 p. 624 .

[6] A.K.A. Hakeem, S. Saranya, B. Ganga, J. Mol. Liq. 230, 445 (2017).

[7] F. Fadaei, A.M Dehkordi, M. Shahrokhi, Z. Abbasi, Appl. Therm. Eng. 116, 329 (2017).

[8] V. Chaudhary, Z. Wang, A. Ray, I. Sridhar, R.V. Ramanujan, J. Phys. Appl. Phys. 50, 03LT03 (2017).

[9] P. Gas, A. Miaskowski, 2015 Selected Problems of Electrical Engineering and Electronics (WZEE) IEEE Xplore 2015, 7394040 (2015).

[10] S.K. Das, S.U.S. Choi, H.E. Patel, Heat Transf. Eng. 27, 3 (2006). 